\title{
Results of 131 consecutive operated patients with a displaced proximal humerus fracture: an analysis with more than two years follow-up
}

\author{
D. Faraj • B. W. Kooistra • W. A. H. vd Stappen • \\ A. J. Werre
}

Received: 31 March 2010/Accepted: 13 May 2010/Published online: 15 June 2010

(c) The Author(s) 2010. This article is published with open access at Springerlink.com

\begin{abstract}
Objective The purpose of this study is to determine the efficacy of the open reduction and fixation of a dislocated proximal humerus fracture with a locking plate, also evaluating the incidence of complications and functional recovery of the shoulder. A retrospective study focusing on patients who had suffered a fracture of the proximal humerus and were treated by means of an open reduction and internal fixation with a locking plate, using either the Philos or the LPHP plate. We have also included a comparison of these two plates.

Method Ninety-two patients were found to be eligible and were included in our study. These patients had all been treated for a proximal humerus fracture between 2002 and 2008. We included those patients who could be classified as a class 2, 3, or 4 according to the Neer classification. Demographic data, surgical technique, and peri/post-operative complications were collected from medical records. Patients were followed-up and the Oxford Shoulder Score (OSS) was filled in by phone. The mean age of our patient population was 66.2 (15-97), with a male: female ratio of 1:5 (15:77). (The median follow-up was 2.4 years $(0.2-$ 5.8). Fourteen of our patients died during follow-up, while 25 patients were otherwise lost to follow-up. For this study, out of the original 131 patients, this finally resulted in 92 patients whom we followed-up post-operatively.

Results Ninety-two patients $(70 \%, 92 / 131)$ were interviewed. The mean Oxford Shoulder Score (OSS) was 19.76
\end{abstract}

D. Faraj $(\bowtie) \cdot$ B. W. Kooistra · W. A. H. vd Stappen .

A. J. Werre

Department of Surgery, Canisius Wilhelmina Hospital, P.O. Box 9015, 6500 GS Nijmegen, The Netherlands

e-mail: D.Faraj3@gmail.com
(11-54). The overall complication rate was 39.1\% (36/92). The most frequently occurring complications in our patient population were hemorrhage $3.3 \%$; dislocation of the caput humeri and/or tuberculum majus 2.2\%; persistent pain $3.3 \%$; Avascular necrosis (AVN) of the humeral head 0\%; Loss of reduction and screw cutout $6.5 \%$; Plate breakout 6.5\%; Subacromial Impingement 11.9\%; Frozen shoulder $3.3 \%$; rotator cuff rupture $1.1 \%$, and infection $1.1 \%$. Of the entire group of patients originally included in this study, $29 \%$ was re-operated due to one of the above-mentioned complications.

Conclusion In conclusion, the locking plate provides satisfactory functional outcomes after a mid-term followup in patients with displaced proximal humerus fractures. The incidence of complications and subsequent re-operation is relatively high, however, comparable to or slightly better when compared to data found in literature. Subacromial Impingement seems to occur more frequently when a Philos plate is implemented. We therefore suggest that randomized clinical trials determining the possible superiority of one specific type of plate in patients with a displaced proximal humerus fracture are to be performed in future.

Keywords Proximal humerus fractures - LPHP . Oxford shoulder score $\cdot$ Complications

\section{Introduction}

Proximal humerus fractures represent $4-5 \%$ of all fractures and $45 \%$ of all humerus fractures [1-5]. The proximal humerus fracture is associated with osteoporosis and mostly affects the elderly. It is the third most common fracture after hip and distal radius fractures, in people 
above 65 years of age [6]. However, proximal humerus fractures do also occur in young people, oftentimes as the result of a high-energy trauma or accident.

The extent of dislocation is an essential factor with regard to the choice of treatment. Non-displaced or minimally displaced fractures can be treated conservatively. Displaced fractures of the humerus are preferably treated by means of surgical intervention, specifically an open reduction and internal fixation $[2,7,8]$.

A variety of techniques have been described as possible treatment of the proximal humerus fracture. Due to the high complication rates as reported by both Thalhammer et al. and Szyszkowitz et al. [9, 10], it remains uncertain which of these is the optimal treatment of the proximal humerus fracture.

During the last decade, many new devices have been developed to aid in the treatment of the dislocated proximal humerus fracture. In particular, the locked screw-plate devices allow for improved fixation in osteoporotic bone $[11,12]$. Because of the enhanced stability that these devises offer, early mobilization can be achieved. Application of these plates through a minimally invasive technique provides a further great advantage, namely the prevention of additional trauma to the soft tissue [13]. However, there are few studies available that actually evaluate the results of this technique or report on the treatment-related complications.

The aim of this study was to determine the efficacy of fixation of both the LPHP ${ }^{\circledR}$ and Philos ${ }^{\circledR}$ plate (Synthes, Oberdorf, Switzerland), as well as the subsequent complication rates and the functional recovery of the shoulder in patients who underwent surgery for a displaced proximal humerus fracture at our hospital.

\section{Patients and methods}

We commenced our study by selecting one hundred and thirty-one patients who had been surgically treated for a displaced proximal humerus fracture between January 2002 and December 2008. All patients were classified according to the Neer classification [14]. Patients with type 2, 3, or 4 proximal humerus fractures were included in our study. Patients with a previous ipsilateral fracture were excluded.

All patients underwent surgery, and either the Locked Proximal Humerus Plate (LPHP) or a Philos plate (Synthes ${ }^{\circledR}$, Oberdorf, Switzerland) was used to fixate the fracture. For most patients, an anterior approach was preferable; however, in a few patients, the surgeon decided upon a lateral deltoid-splitting approach. Open reduction of the main fracture and the greater tuberosity was achieved in all cases. Subsequently, the plate was inserted and positioned approximately one centimeter below the upper edge of the greater tuberosity in order to avoid post-operative subacromial impingement (SAI). The reduction was temporarily fixed with $\mathrm{K}$-wires and verified by means of an image intensifier. Once a satisfactory anatomic reduction was achieved, the $\mathrm{K}$-wires were replaced by locking screws. In case of a tear or avulsion, the rotator cuff and the subscapularis muscle were repaired.

All patients were invited to visit our outpatient clinic for regular checkups post-operatively. Demographic data, surgical technique, peri- and post-operative complications were collected from our patients' medical records. After obtaining informed consent from all participating patients, we commenced our telephone interviews with patients at their convenience. The primary end point was the Oxford Shoulder Score (OSS). The level of pain was scored using the validated VAS-score. The OSS ranges from 10 to 60 points, with the higher scores representing a worse functional outcome [15]. Secondary end points were early post-operative complications such as infection or hemorrhage. Complications that occurred after some time were, among others, broken (distal and proximal) plate or screw failures, vascular necrosis of the head, plate displacement, screw protrusion, rotator cuff rupture, screw migration, and SAI.

\section{Results}

The mean age of our ninety-two patients was 66.2 (15-97), with a male: female ratio of 1:5 (15:77). Out of these 131 patients, 14 patients passed away during the follow-up period. Twenty-five patients were lost to follow-up or were otherwise unavailable to participate in the interview. All of the remaining 92 patients were included and interviewed by telephone. This resulted in a follow-up rate of $70 \%$.

Prior to initial surgery, the majority of patients were classified as Neer III $(n=74$, to follow-up $=55)$, while 29 (to follow-up $=19$ ) patients were classified as Neer II and 28 patients (to follow-up $=18$ ) belong to the Neer IV category (Table 1). During the six-year time span of this study, two types of locking plates were implanted (Table 2). Fifty-five patients underwent surgery receiving a LPHP device; more specifically, 46 patients received a 5-hole LPHP plate, and in 9 patients, an 8-hole LPHP plate

Table 1 Characteristics according to Neer classification

\begin{tabular}{lllcc}
\hline & $\begin{array}{l}\text { Patients to } \\
\text { follow-up }\end{array}$ & $\begin{array}{l}\text { Function } \\
\text { (Mean OSS) }\end{array}$ & Complications & Re-operations \\
\hline Neer II & 19 & 18.7 & $9(9.8 \%)$ & $7(7.6 \%)$ \\
Neer III & 55 & 20.1 & $17(18.5 \%)$ & $14(15.2 \%)$ \\
Neer IV & 18 & 19.9 & $9(9.8 \%)$ & $6(6.5 \%)$ \\
\hline
\end{tabular}


Table 2 Characteristics according to type of device used

\begin{tabular}{lllll}
\hline & $\begin{array}{l}\text { Patients to } \\
\text { follow-up }\end{array}$ & $\begin{array}{l}\text { Function } \\
\text { (Mean OSS) }\end{array}$ & Complications & Re-operations \\
\hline LPHP & 55 & 17.4 & $22(23.9 \%)$ & $17 / 55(30.9 \%)$ \\
Philos & 37 & 23.9 & $14(15.2 \%)$ & $10 / 37(27 \%)$ \\
\hline
\end{tabular}
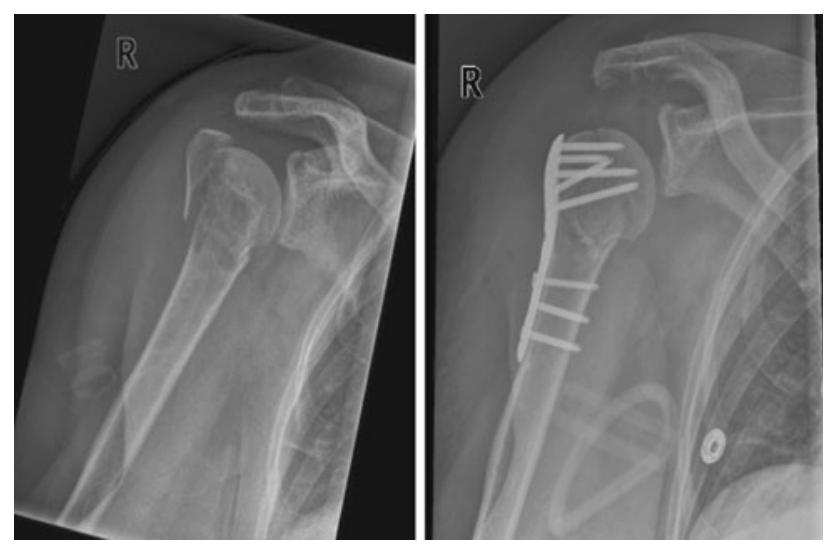

Fig. 1 Before and after surgery with a 3-hole Philos plate

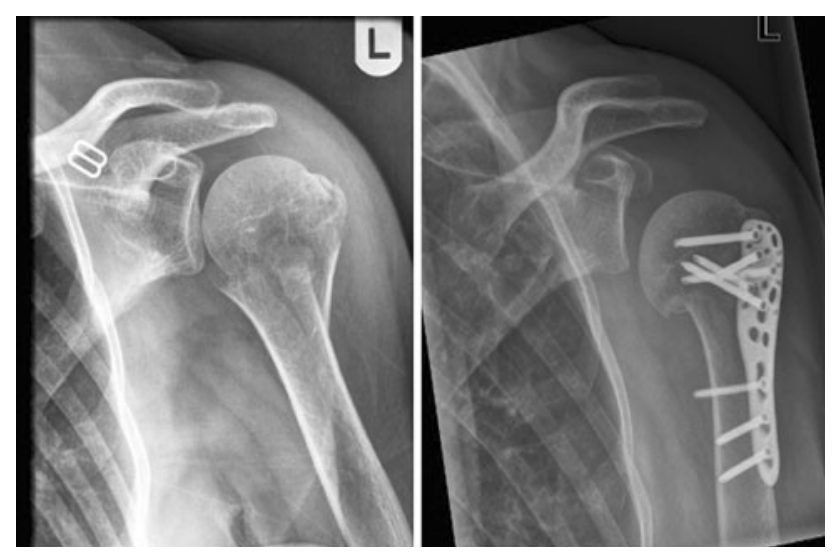

Fig. 2 Before and after surgery with a 3-hole Philos plate

was implanted. The remaining 37 patients were treated with a Philos plate (a 3-hole plate) (Figs. 1, 2, and 3).

After a mean follow-up of 2.4 years (range: 0.2-5.8), the mean pain score according to the VAS-score was 2.9 (range: 0-10). The median oxford shoulder score was 19 (range: 11-54). Various complications were registered (Table 3). Three patients suffered hemorrhaging for which they were treated conservatively. One patient suffered a dislocation of the caput humeri and/or tuberculum majus, necessitating revision surgery. Three other patients experienced persistent pain necessitating renewed surgical intervention. In these 3 patients, the plate was removed one year after the initial operation. Avascular necrosis (AVN) did not occur in this study. Loss of reduction and

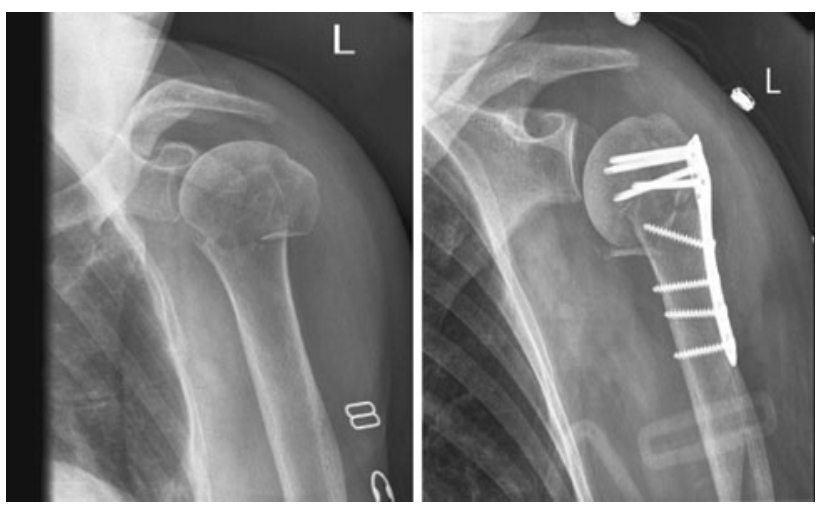

Fig. 3 Before and after surgery with a 5-hole LPHP

screw cutout occurred in six patients. Plate breakage occurred in six patients (one proximal and 5 distal) (Fig. 4). Five of these patients had received a LPHP plate, and a broken Philos plate was reported in only one patient. Eleven patients subsequently suffered from a subacromial impingement. Eight of these patients had had a Philos plate implanted, whereas only three patients had received the LPHP plate. This difference is of statistic significance $(P=0.019)$. Frozen shoulder occurred in three patients. Only one patient had a clinically diagnosed rotator cuff rupture. This results in a total complication rate of $39.1 \%$ (36/92). Out of these 36 patients, 22 patients belonged to the LPHP group. Due to these complications, $29.3 \%$ (27/92) of all patients underwent a second operation.

\section{Discussion}

As mentioned earlier, the mean age of our study group was 67 (15-100). This explains the high incidence of osteoporosis in these elderly patients. These displaced proximal humerus fractures were mainly due to a low-energetic trauma such as a fall onto an outstretched arm. This type of fracture resulting from the poor quality of bone in the elderly makes surgical treatment a challenging task.

Traditional treatment of osteoporotic proximal humerus fractures with conventional plates and screws leads to high failure rates due to the bone resorption underneath the plate, high rotational forces, and insufficient purchase of the screws in the frequently osteoporotic humeral head [12]. This has led to the development and implementation of the Locking Proximal Humeral Plate. The LPHP is based on the locking compression plate principle and functions as an internal fixation device. It is pre-contoured to fit the lateral proximal humerus and is attached in the head by means of locking screws and locking or compression screws in the shaft $[16,17]$. 
Table 3 Postoperative complications after treatment with LPHP/Philos plate

\begin{tabular}{|c|c|c|c|c|c|}
\hline Complications & $N(\%)$ & LPHP $N=$ & Philos $N=$ & Re-operation $N=$ & $\begin{array}{l}\text { Statistical } \\
\text { significance, } P\end{array}$ \\
\hline Subacromial impingement & $11(11.9 \%)$ & 3 & 8 & 10 & 0.019 \\
\hline Loss of reduction and cutout & $6(6.5 \%)$ & 3 & 3 & 6 & $>0.05$ \\
\hline Plate breakage & $6(6.5 \%)$ & 5 & 1 & 4 & $>0.05$ \\
\hline Persistent pain & $3(3.3 \%)$ & 3 & 0 & 3 & $>0.05$ \\
\hline Hemorrhage & $3(3.3 \%)$ & 3 & 0 & 0 & $>0.05$ \\
\hline Head/tuberculum majus slip off (Malalignment) \& shoulder luxation & $2(2.2 \%)$ & 1 & 1 & 1 & $>0.05$ \\
\hline Frozen shoulder & $3(3.3 \%)$ & 3 & 0 & 2 & $>0.05$ \\
\hline Infections & $1(1.1 \%)$ & 0 & 1 & $0^{*}$ & $>0.05$ \\
\hline Rotator cuff rupture & $1(1.1 \%)$ & 1 & 0 & 1 & $>0.05$ \\
\hline Avascular humerus head necrosis & $0(0 \%)$ & - & - & - & - \\
\hline Total & $36(39.1 \%)$ & 22 & 14 & $27(29.3 \%)$ & \\
\hline
\end{tabular}

* This patient suffered a plate breakage as well (re-operated for this reason)

To the best of our knowledge, this is one of the few studies focusing on the treatment of proximal humerus fractures by means of a locking plate, with a follow-up of patients over a two-year period (mean 29 months). The recently published systematic review by Thanasas et al. [18] included a total of twelve studies. Only one of these studies achieved a mean follow-up of 31 months, the other 11 of these studies had a mean follow-up of approximately 12 months.

Avascular necrosis (AVN) is one of the most dramatic complications requiring re-operation. Fortunately, none of the patients in our study experienced this complication, which necessitates renewed surgery and replacement of the humeral head. This is an excellent result when compared with similar studies in published literature, e.g., Agudelo et al. reported an avascular necrosis rate of $4.5 \%$ [12]. The systematic review by Thanasas et al. reported an even higher rate of AVN, namely $7.9 \%$ [18]. We have no clear explanation for this significant difference, perhaps performing surgery within $24 \mathrm{~h}$ and the use of fewer screws in the humeral head has resulted in a preventative effect toward the occurrence of AVN.

Within our patient population, loss of reduction and screw cutout occurred in six $(6.5 \%)$ patients (5 proximal, 1 distal). All six patients were re-operated due to this complication. This percentage is lower than the $31 \%$ found in published data [18]. Three $(3.3 \%)$ additional patients complained of chronic pain and were successfully re-operated; their locking plates were surgically removed.

Plate breakage occurred in six (5 LPHP, 1 Philos) patients $(6.5 \%)$. Four patients required renewed surgery. Re-operation consisted of renewed fixation through cerclage or replacing the broken plate with a longer LCP (low contact plate) or Philos plate. Our percentage of plate breakage is higher than the percentages found in published literature. Thanasas et al. [18] reported this complication to be rare with an incidence of $0.7 \%$. A possible explanation for the rather high rate of plate breakage could be the high incidence of osteoporotic bone in our study group, resulting in a higher incidence of comminution. It is known that the plate breaks at the level of the fracture when severe comminution of the medial cortex exists [16, 18]. Also, poor reduction could lead to plate breakage due to fatigue failure $[18,19]$. Another possible explanation for our high rate of hardware failure could be related to physiotherapy. By raising the operated arm without any external support, an extraordinary force could affect the plate leading to fatigue and breakage. We have therefore recently changed our physiotherapy protocol. Changing the manner of mobilizing the arm in order to circumvent this as a possible cause for breakage. Additionally, the LPHP may be weaker than the Philos plate resulting in a higher rate of plate breakage (Breakage: LPHP 5, Philos 1). This difference was not statistically significant $(P=0.228)$. Nowadays, only the Philos plate is used in our hospital.

Only one patient suffered an infection post-operatively. This patient who suffered a low-grade infection was treated conservatively; however, the same patient required renewed surgery in a later stadium due to plate breakage. This amounts to an overall infection rate of $1.1 \%$, a rate that is consistent with the infection rates reported in literature. Brunner et al. [20] reported an infection rate of $2 \%$. Agudelo et al. [12] published a rate of $4.5 \%$.

The soft tissue complications were subacromial impingement (SAI), rotator cuff rupture, and frozen shoulder. In our patient population, $11.9 \%$ (11/92) were diagnosed with SAI. Ten of these eleven patients (8 Philos, 3 LPHP) were re-operated due to this complication. Siwach et al. [21] reported a similar rate of $8 \%$ in their study. The systematic review of twelve studies by Thanasas et al. [18] 


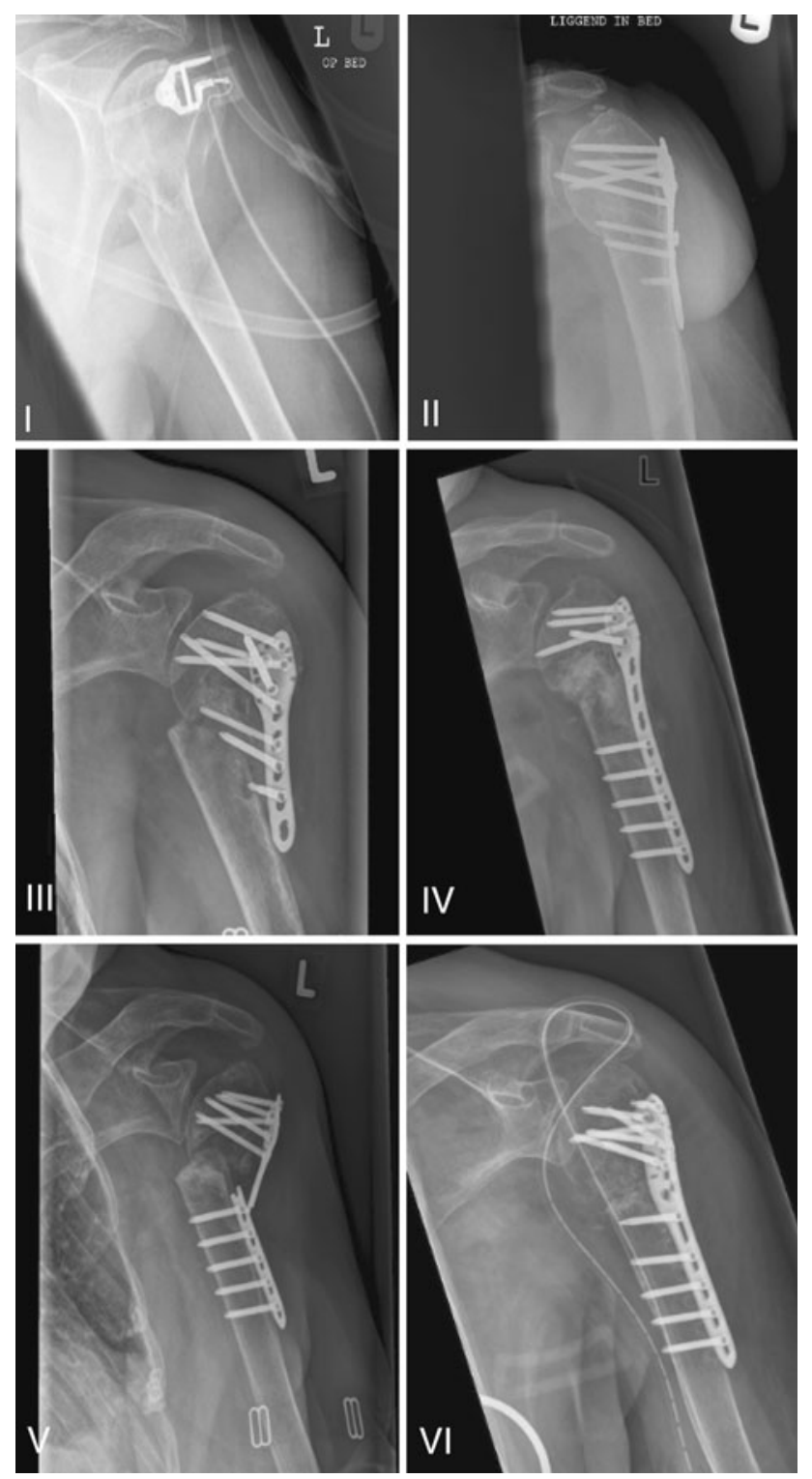

Fig. 4 A case of a patient with a plate breakage as complication. I: proximal humerus fracture. II: 5-hole LPHP. III: loss of reduction with the 5-hole LPHP. IV: re-operation using a 8-hole LPHP. V: Breakage of the 8-hole LPHP. VI: re-operation using a Philos plate

reported an impingement rate of 5.5\%. Only one patient experienced rotator cuff rupture and tendinosis necessitating a second operation. This rupture was not obvious or may be not identified preoperatively. One year after surgery, a minimal rupture of the biceps muscle was diagnosed with an ultrasound. Two out of the three patients with a frozen shoulder were treated surgically, removing the implanted hardware/locking plates. This represents a percentage of $2 \%$, also consistent with the percentages reported in literature. [12].

The functional outcome following surgical intervention and insertion of a locking plate was determined using the
Oxford shoulder score (OSS). The OSS ranges from 10 to 60 points, with the higher scores representing the worst functional outcome. After 2.4 years, the median OSS was $19.76(11-54)$. This is a relatively low score on a maximum scale of 60 . A search through the majority of published articles reveals that most studies made use of the Constant score [22] in order to assess shoulder function. This scoring tool consists of four parameters that are used to assess the function of the shoulder: including pain, daily activity (sleep, work, recreation/sport), range of motion, and strength. The minimum score is 0 and the maximum score is 100. A low score means a worse functional outcome, while a higher score is correlated with better shoulder function. Our motivation to use the OSS instead of the Constant score was the fact that we decided to collect our data by means of a telephone interview. Considering the relatively high mean age of our patient population, we felt that a telephone interview would be preferable to extending an invitation to our outpatient clinic, which would have necessitated travel. The systematic review by Thanasis et al. [18] reported an overall Constant score of 74.3. Compared to the OSS score, this means a score of 15 points [1]. The level of pain according to the VAS-score was 2.9 on a scale of 0-10. Fankhauser et al. [16] reported a pain score of 13.9 on a scale of 15 (Pain score of the Constant score system). Hente et al. [23] and Voigt et al. [24] reported a pain score of 11 and 12 , respectively.

In conclusion, we find after a mid-term follow-up that the locking plate provides satisfactory functional outcomes in patients who have sustained a displaced proximal humerus fracture. The complication and re-operation rate is relatively high, however, comparable to or slightly better than the rates reported in published literature. Although subacromial impingement seems to occur more frequently when a Philos plate is implemented, we recommend that carefully executed randomized clinical trials should be performed to determine whether the use of one type of plate is actually superior or preferable to the other in achieving fixation of a displaced proximal humerus fracture.

Conflict of interest statement No benefits in any form have been or will be received from a commercial party related directly or indirectly to the subject of this manuscript.

Open Access This article is distributed under the terms of the Creative Commons Attribution Noncommercial License which permits any noncommercial use, distribution, and reproduction in any medium, provided the original author(s) and source are credited.

\section{References}

1. Baker P, Nanda R, Goodchild L, Finn P, Rangan A (2008) A comparison of the constant and Oxford shoulder scores in patients 
with conservatively treated proximal humeral fractures. J Shoulder Elbow Surg 17(1):37-41

2. Zyto K, Ahrengart L, Sperber A, Törnkvist H (1997) Treatment of displaced proximal humeral fractures in elderly patients. J Bone Joint surg [Br] 79(3):412-417

3. Horak J, Nilsson BE (1975) Epidemiology of fracture of the upper end of the humerus. Clin Orthop 112:250-253

4. Lind T, Kroner K, Jensen J (1989) The epidemiology of fractures of the proximal humerus. Arch Orthop Trauma Surg 108:285-287

5. Rose SH, Melton U, Morrey BF (1982) Epidemiologic features of proximal humeral fractures. Clin Orthop Relat Res 168:24-30

6. Baron JA, Barrett JA, Karagas MR (1996) The epidemiology of peripheral fractures. Bone 18(3 suppl):209S-213s

7. Gaebler C, McQueen MM, Court-Browm CM (2003) Minimaly displaced proximal humeral fractures: epidemiology and outcome in 507 cases. Acta orthop Scand 74:580-585

8. Koval KJ, Gallagher MA, Marisicano JG, Cuomo F, McShinawy A, Zuckerman JD (1997) Functional outcome after minimally displaced fractures of the proximal part of the humerus. J Bone Joint Surg Am 85:1647-1655

9. Thalhammer G, Platzer P, Oberleitner G, Fialka C, Greitbauer M, Vécsei V (2009) Angular fixation of proximal humeral fractures. J Trauma 66(1):204-210

10. Szyszkowitz R, Seggl W, Schleifer P, Cundy PJ (1993) Proximal humeral fractures. Management techniques and expected results. Clin Orthop Relat Res 292:13-25

11. Sommer C, Gautier E, Muller M, Helfet DL, Wagner M (2003) First clinical results of the locking compression plate (LCP). Injury 34:B43-B54

12. Agudelo J, Schürmann M, Stahel P, Helwig P, Morgan SJ, Zechel W, Bahrs C, Parekh A, Ziran B, Williams A, Smith W (2007) Analysis of efficacy and failure in proximal humerus fractures treated with locking plates. Orthopedics 21(10):676-681

13. Helmy N, Hintermann B (2006) New trends in the treatment of proximal humerus fractures. Clin Orthop 442:100-108
14. Neer CS 2nd (1970) Displaced proximal humeral fractures. I. Classification and evaluation. J Bone Joint Surg Am 52(6):10771089

15. Dawson J, Fitzpatrick R, Carr A (1996) Questionnaire on the perceptions of patients about shoulder surgery. J Bone Joint Surg Br 78(4):593-600

16. Fankhauser F, Boldin C, Schippinger G, Haunschmid C, Szyszkowitz R (2005) A new locking plate for unstable fractures of the proximal humerus. Clin Orthop Relat Res 430:176-181

17. Frigg R (2001) Locking compression plate (LCP). An osteosynthesis plate based on the dynamic compression plate and the point contact fixator (PC-Fix). Injury 32(Suppl 2):63-66

18. Thanasas C, Kontakis G, Angoules A, Limb D, Giannoudis P (2009) Treatment of proximal humerus fractures with locking plates: a systematic review. Shoulder Elbow Surg 18(6):837-844

19. Gautier E, Sommer C (2003) Guidelines for the clinical application of the LCP. Injury 34(Suppl 2):B63-B76 (Review)

20. Brunner F, Sommer C, Bahrs C, Heuwinkel R, Hafner C, Rillmann P, Kohut G, Ekelund A, Muller M, Audigé L, Babst R (2009) Open reduction and internal fixation of proximal humerus fractures using a proximal humeral locked plate: a prospective multicenter analysis. J Orthop Trauma 23(3):163-172

21. Siwach R, Singh R, Rohilla RK, Kadian VS, Sangwan SS, Dhanda M (2008) Internal fixation of proximal humeral fractures with locking proximal humeral plate (LPHP) in elderly patients with osteoporosis. J Orthop Traumatol 9(3):149-153

22. Constant CR, Murley AH (1987) A clinical method of functional assessment of the shoulder. Clin Orthop Relat Res 214:160-164

23. Hente R, Kampshoff J, Kinner B, Füchtmeier B, Nerlich M (2004) Treatment of dislocated 3- and 4-part fractures of the proximal humerus with an angle-stabilizing fixation plate. Unfallchirurg 107(9):769-782

24. Voigt C, Woltmann A, Partenheimer A, Lill H (2007) Management of complications after angularly stable locking proximal humerus plate fixation. Chirurg 78(1):40-46 